\title{
明矾的宏观晶体形状
}

张天一, 潘玛璠, 徐一寒, 崔爱莉 ${ }^{*}$

清华大学化学系, 北京 100084

摘要: 通过简易的热力学模型推测晶体宏观形状及缺陷的成因, 借助于本科无机探究实验, 培养明矾大晶体并测量 其宏观参数, 拟合出明矾的热力学参数, 以解释明矾的宏观形状、缺陷并预测明矾的完美八面体的可能性与晶体的 自范性。

关键词: 明矶; 宏观晶面; 自范性

中图分类号: G64; O6

\section{Macroscopic Morphology of Alum(K) Crystal}

Tianyi Zhang, Yufan Pan, Yihan Xu, Aili Cui *

Department of Chemistry, Tsinghua University, Beijing 100084, P. R .China.

Abstract: In this paper, a simple thermodynamic model of crystal macroscopic morphology and the cause of the defects were suggested. By virtue of growing alum $(K)$ crystals and measuring their macroscopic parameters in the inorganic laboratory for undergraduates, we can calculate the thermodynamic parameters of alum, thus explaining the macroscopic morphology and defect of alum $(\mathrm{K})$, and predicting the possibility for alum $(\mathrm{K})$ to generate octahedral crystals and its self-normality.

Key Words: Alum(K); Macroscopic crystallographic planes; Self-normality

不同的晶体具有不同的宏观形状，如理想的胆矾晶体是平行六面体，而理想的明矾晶体为正八 面体。但在培养过程中，晶体常出现截棱或截角的六面体、八面体。本文在 Bravais 原理的基础上， 对晶体的宏观形状及缺陷提出简单的热力学假设, 并通过明矾培养实验对理论进行半定量的解释, 希望预测明矾的宏观形状、缺陷及自范性等性质。

\section{1 宏观晶体形状的探究}

晶体的宏观形状决定于晶体的微观结构, 而晶体的物理性质的对称性经常具有比晶体所属的点 群更高的对称性 ${ }^{[1]}$ 。对称性在晶体学中有广泛的应用, 包括在光学、电磁学上的对称性等, 而此类对 称性皆源于结构上的对称性。在本文中, 我们将对称性狭义地解释为几何上的对称性, 其最显著的 宏观表现即为宏观晶体形状的对称性。

对于晶体的宏观对称性, Bravais 曾做出解释: 晶体上的实际晶面平行于面网密度大的面网, 且 面网密度越大, 相应的晶面的重要性也越大 ${ }^{[2]}$ 。这是由于若将晶体当作二维晶体的堆积, 则二维晶 体的面密度 $\mu$ 与层间距 $d$ 的比值为晶体密度: 
即:

$$
\rho=\mu / d
$$

$d \propto \mu$

而晶面解离能与层间距一般负相关, 二维晶体的面密度与面网密度正相关, 故面网密度愈大, 愈易 解离。

二维晶体的面密度直接决定于晶体结构与晶面指标, 且其对称性不小于晶体(微观)对称性, 所 以我们能理解晶体的宏观对称性不小于其微观对称性。但截棱与截角亦不破坏宏观对称性, Bravais 的理论对此则无法进行有力解释。

晶体宏观生长的理论很多, 但多数既不普适, 亦不简洁。在此, 我们仅考虑晶体的表面能与内 部能, 以半实验半理论的方式半定量地解释晶体宏观结构。

首先我们以热力学的观点重新审视 Bravais 法则:

$$
E=\alpha V+\Sigma \beta_{i} S_{i}
$$

式中 $E$ 为晶体总能量, $V$ 为晶体体积, $S_{i}$ 表示某特定取向的宏观晶面面积, $\alpha 、 \beta_{i}$ 为单位体积能和某 特定取向的宏观晶面的单位表面能。

一般认为单位体积能 $\alpha$ 为负, 单位表面能 $\beta$ 为正, 面网密度最大的面作为暴露面, 其单位表面 能最小。在其他条件相同的情况下, 晶体总能量最低, 最稳定。

而截棱或截角, 则是让较不稳定的面暴露, 即单位表面能升高, 且其体积减小使内部的稳定化 作用降低。但由此带来的是特定取向暴露面的面积减小, 若因为面积减小所降低的能量能弥补单位 表面能增加及体积减小所升高的能量, 晶体将可能出现截棱(或截角)的情况。

当然, 由于动力学原因(如较高的能垒), 晶体不一定能达到所有可能状态中能量的最小值。但对 于处于稳态的晶体, 我们有理由认为其处于能量的极小值, 即:

$$
\mathrm{d} E=0
$$

由此, 可在测出稳态晶体的一些参数后, 计算得 $\alpha 、 \beta$ 等, 以解释或预测晶体的宏观几何形状。

\section{2 实验过程}

\section{1 选材}

本科无机实验中要求的是胆矾晶体的培养, 但如果以胆矾为实验材料, 则实验结果较难处理。 胆矾属 $P$ 群, 为三斜晶系(图 1 左), 参数多, 体积、面积函数较为复杂。若考虑所有截棱、截角情 况, 共有体积、 3 组平行六面体面的参数、 6 组棱的参数、 4 组顶点的参数共计 14 个参数; 仅考虑 主要晶面[100]、[010]、[001] (平行六面体的面)和次要晶面[111]、[120]、[121]和[021] (平行六面体的 棱或角) ${ }^{[2]}$ 亦有 8 个参数; 其体积面积函数亦涉及到繁杂的三角比。

而胆矾难以处理的根本原因，在于其过低的对称性，若是选择对称性较高的明矾作为实验材料， 则将大有改观。明矾晶体属Pa3 群, 属立方晶系(图 1 右), 从其对称性出发, 所有的棱、角、面是等 价的, 故仅有体积参数、全同的正八面体的面的参数、棱的参数和顶点的参数共计 4 个参数, 而其 体积、面积函数亦极为简便, 是较为理想的选材。

\section{2 晶体培养}

\subsection{1 实验原理}

用胆矾的结晶法(悬线法将晶种置于饱和溶液中, 用带孔保鲜膜封住烧杯使溶液缓慢挥发溶剂) 培养明矾晶体较难控制, 其原因包括: 明矾在高温下溶解度极大, 而析晶速度慢, 故容易配成过饱 和度较大的过饱和溶液, 使析晶多而碎, 达不到生长完美大晶体的目的; 溶剂缓慢蒸发时在表面的 溶液浓度高而下层浓度低, 在静置条件下较难确保母液的均匀性, 可能出现母液表面析出晶膜但晶 种并不长大的情况。故在本实验中不选择饱和溶液的溶剂挥发法培养明矾大晶体。

查得明矾在水中于不同的温度下溶解度如表 1 所示。

为使晶体生长的速率提高, 本实验将直接以明矾的均匀过饱和溶液 (热饱和溶液的过冷溶液)作 为母液, 保证单位质量的水中溶解的明矾超过其平衡溶解度。 


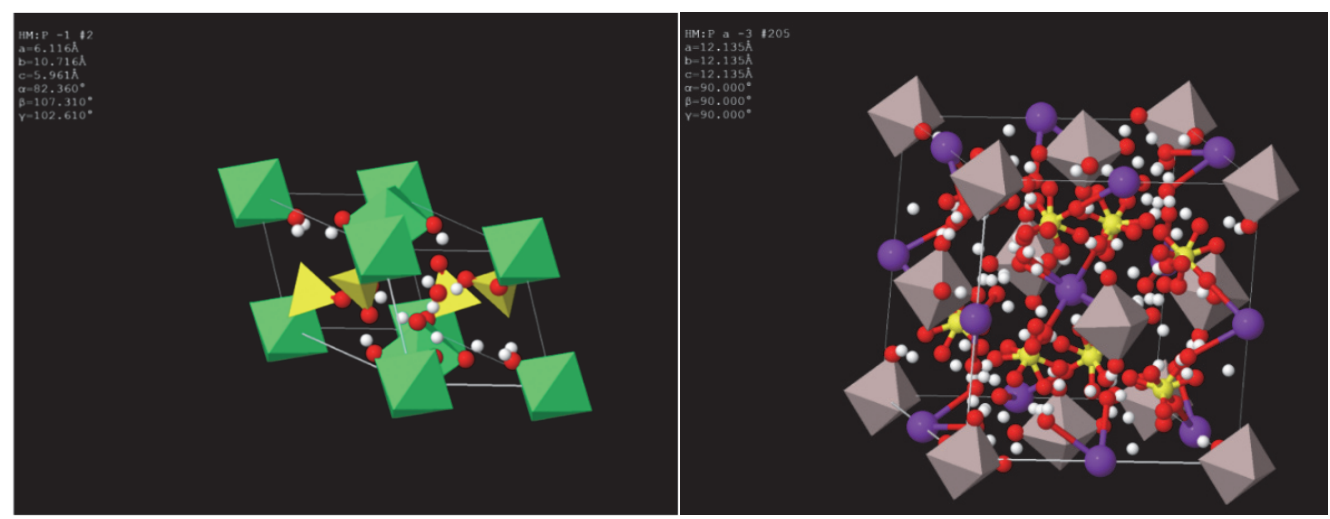

图 1 胆矾晶胞(左)与明矾晶胞(右) (图源自 ICSD ${ }^{[3]}$ )

表 1 明矾的溶解度

\begin{tabular}{cc||cc}
\hline 温度 $/{ }^{\circ} \mathrm{C}$ & 溶解度 $/(\mathrm{g} / 100 \mathrm{~g}$ 水 $)$ & 温度 $/{ }^{\circ} \mathrm{C}$ & 溶解度 $/(\mathrm{g} / 100 \mathrm{~g}$ 水 $)$ \\
\hline 0 & 3 & 60 & 25 \\
10 & 4 & 70 & 40 \\
20 & 5.9 & 80 & 71 \\
40 & 11.7 & 90 & 109 \\
50 & 17 & 100 & 154 \\
\hline
\end{tabular}

溶液的过饱和度 $\sigma=\left(c-c_{0}\right) / c_{0}=m / m_{0}$, 式中 $m$ 为向饱和溶液中补充的明矾质量, $m_{0}$ 为饱和溶液 中原本的明矾质量。在实验操作中, 每次生长晶体之前会将大晶体暂时取出母液, 加热溶解母液中 析出的碎晶并适量补充明矾粉末。若不考虑溶剂挥发(实际操作中为保持液面在确定的刻度线, 必要 时将补充溶剂), 母液系统唯一损失的明矾即大晶体生长的部分, 为保证溶液的过饱和度保持在一定 范围内，每次补加的明矶量即大晶体生长质量。

\subsection{2 实验原理}

晶种的培养:

1) 配制过饱和母液 $20 \mathrm{~g} / 100 \mathrm{~g}$ 水 $100 \mathrm{~mL}$, 加热搅拌至溶解(约 $60^{\circ} \mathrm{C}$ 溶解), 悬线静置, 过夜。 线上析出透明晶种, 杯底有碎晶析出;

2) 在线上选取完美晶种备用。

晶体的生长:

1) 取出母液中晶种或晶体, 母液与烧杯底部碎晶加热搅拌溶解, 冷却备用。若底层析晶少(即晶 体成长较快), 可适量补充明矾粉末;

2) 将晶体悬吊放入过饱和母液, 放置过夜。过饱和的明矾在晶体表面析出, 晶体生长, 溶液饱 和，杯底析晶;

3) 重复 “晶体的生长” 中 1)、2)操作若干次, 得较大的晶体, 在尚未长成完美八面体之前, 量 取晶体长度。

经实验发现, 明矾生长过程中并未出现截棱的情况, 为截角八面体(图 2、图 3), 故只有体积、 八面体面 $(8$ 个, 六边形, 总面积 $S 1$ )与截角面 $(6$ 个, 正方形, 总面积 $S 2)$ 相关的参数须处理。

\section{3 数据处理}

测量图 4 中的 $a 、 b$ 长度, 其中 $a$ 为完美八面体晶体棱长, $b$ 为截角面正方形边长。通过 $a 、 b$ 可 求得晶体的体积、表面积等物理量, 结合设定的单位表面能和单位体积能等热力学参数可计算得晶 体的总能量(仅考虑体积能和表面能)。而稳定的晶体势必处于能量的极小值上, 即 $\mathrm{d} E=0$, 由此可拟 合出单位表面能与单位体积能。记录数据如表 2 所示，精确至 $0.5 \mathrm{~mm}$ 。 


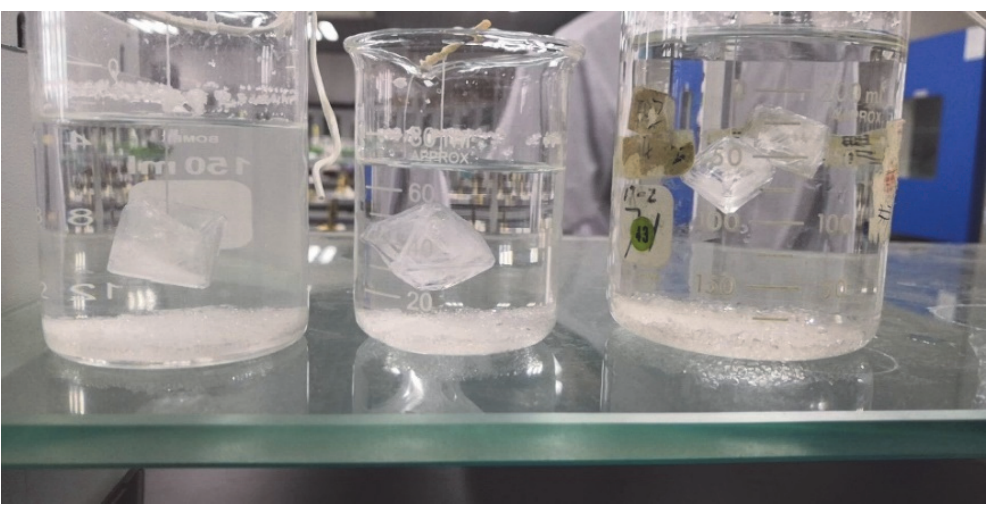

图 2 培养出的明矾大晶体照片

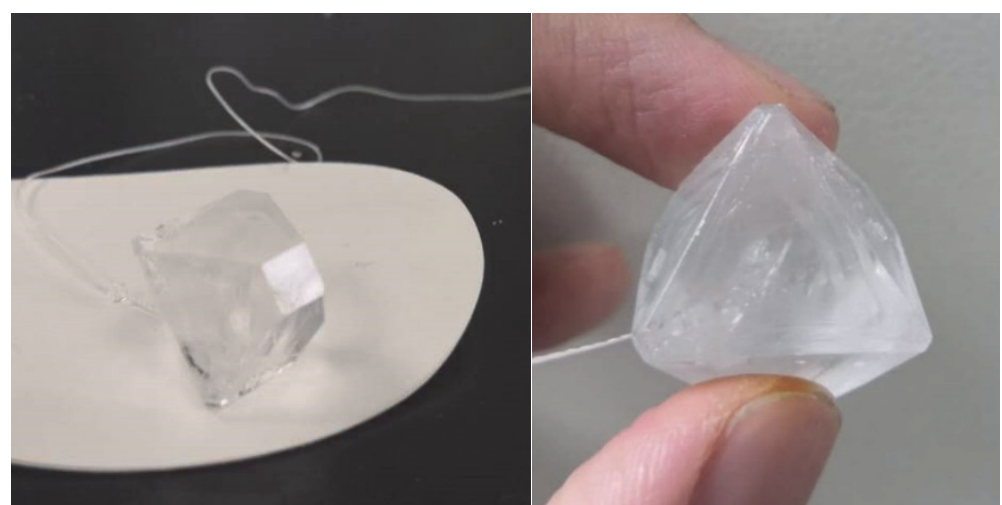

图 3 截角八面体(左)与完美八面体(右)
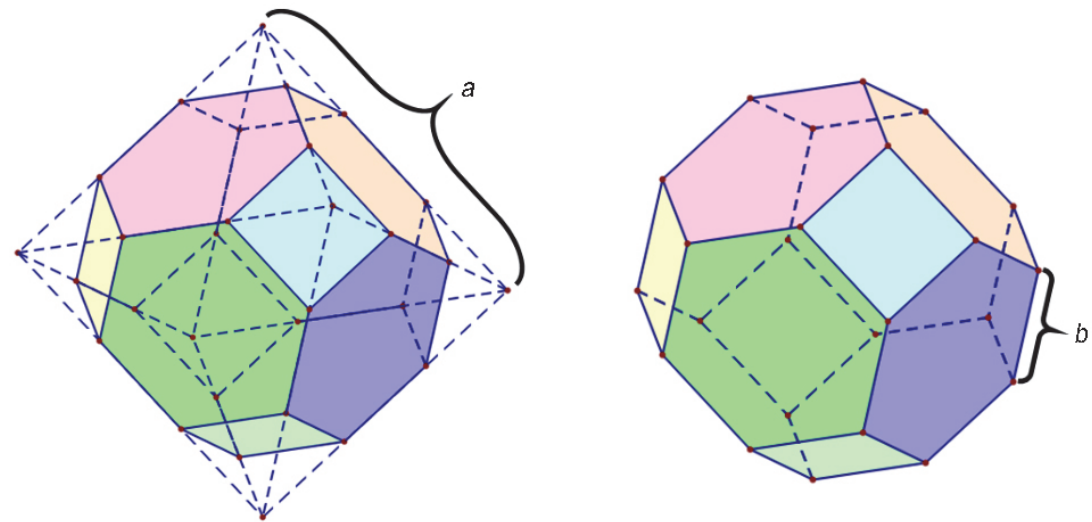

图 4 测量值 $a(\mathrm{~cm})$ (左)与测量值 $b(\mathrm{~cm})$ (右)

\begin{tabular}{ccc} 
表 2 & 明矾大晶体 $(\boldsymbol{a}, \boldsymbol{b})$ 的测量值 \\
\hline 编号 & $a / \mathrm{cm}$ & $b / \mathrm{cm}$ \\
\hline 1 & $0.90(5)$ & $0.10(5)$ \\
2 & $1.30(5)$ & $0.30(5)$ \\
3 & $1.50(5)$ & $0.40(5)$ \\
4 & $1.60(5)$ & $0.45(5)$ \\
5 & $1.70(5)$ & $0.50(5)$ \\
\hline
\end{tabular}


数学建模过程如下:

$$
\begin{aligned}
& E=\alpha V+\beta_{1} S_{1}+\beta_{2} S_{2} \\
& V=\frac{\sqrt{2}}{3} a^{3}-\sqrt{2} b^{3} \\
& S_{1}=8\left(\frac{\sqrt{3}}{4} a^{2}-\frac{3 \sqrt{3}}{4} b^{2}\right)=2 \sqrt{3} a^{2}-6 \sqrt{3} b^{2} \\
& S_{2}=6 b^{2}
\end{aligned}
$$

设在晶体表面各处增厚 $\mathrm{d} l$, 则体积、各表面积将发生变化, 使总能量发生变化:

$$
\begin{aligned}
& \mathrm{d} a=\sqrt{6} \mathrm{~d} l \\
& \mathrm{~d} b=\mathrm{d} a-\sqrt{2} \mathrm{~d} l=(\sqrt{6}-\sqrt{2}) \mathrm{d} l \\
& \mathrm{~d} V=\sqrt{2} a^{2} \mathrm{~d} a-3 \sqrt{2} b^{2} \mathrm{~d} b=\left(2 \sqrt{3} a^{2}-(6 \sqrt{3}-6) b^{2}\right) \mathrm{d} l \\
& \mathrm{~d} S_{1}=4 \sqrt{3} a \mathrm{~d} a-12 \sqrt{3} b \mathrm{~d} b=(12 \sqrt{2} a-(36 \sqrt{2}-12 \sqrt{6}) b) \mathrm{d} l \\
& \mathrm{~d} S_{2}=12 b \mathrm{~d} b=((12 \sqrt{6}-12 \sqrt{2}) b) \mathrm{d} l \\
& \mathrm{~d} E=\alpha \mathrm{d} V+\beta_{1} \mathrm{~d} S_{1}+\beta_{2} \mathrm{~d} S_{2} \\
& \frac{\mathrm{d} E}{\mathrm{~d} l}=\left(2 \sqrt{3} a^{2}-6(\sqrt{3}-1) b^{2}\right) \alpha+(12 \sqrt{2} a-12(3 \sqrt{2}-\sqrt{6}) b) \beta_{1}+(12(\sqrt{6}-\sqrt{2}) b) \beta_{2}
\end{aligned}
$$

又在动力学稳定时, 有:

$$
\frac{\mathrm{d} E}{\mathrm{~d} l}=0
$$

由此, 可通过各组 $(a, b)$ 拟合得 $\frac{\beta_{1}}{\alpha}$ 与 $\frac{\beta_{2}}{\alpha}$, 过程如下:

记 $\frac{\mathrm{d} E}{\mathrm{~d} l}$ 中 $\alpha 、 \beta_{1}$ 与 $\beta_{2}$ 的系数分别为 $k_{0} 、 k_{1} 、 k_{2}$ :

$$
\begin{aligned}
& k_{0}=2 \sqrt{3} a^{2}-(6 \sqrt{3}-6) b^{2} \\
& k_{1}=12 \sqrt{2} a-(36 \sqrt{2}-12 \sqrt{6}) b \\
& k_{2}=(12 \sqrt{6}-12 \sqrt{2}) b
\end{aligned}
$$

代入实验值 $a 、 b$, 计算得 $k_{0} 、 k_{1} 、 k_{2}$, 如表 3 所示。

表 3 明矾大晶体热力学参数的系数

\begin{tabular}{cccccc}
\hline 编号 & $a / \mathrm{cm}$ & $b / \mathrm{cm}$ & $k_{0} / \mathrm{cm}^{2}$ & $k_{1} / \mathrm{cm}$ & $k_{2} / \mathrm{cm}$ \\
\hline 1 & 0.90 & 0.10 & 2.76 & 13.12 & 1.24 \\
2 & 1.30 & 0.30 & 5.46 & 15.61 & 3.73 \\
3 & 1.50 & 0.40 & 7.09 & 16.85 & 4.97 \\
4 & 1.60 & 0.45 & 7.98 & 17.47 & 5.59 \\
5 & 1.70 & 0.50 & 8.91 & 18.09 & 6.21 \\
\hline
\end{tabular}

回归得:

$$
0.81 k_{0}+11.01=k_{1}=k_{2}+11.88
$$

即:

$$
\left\{\begin{array}{l}
\frac{\beta_{1}}{\alpha}=-0.09 \\
\frac{\beta_{2}}{\alpha}=-1.14
\end{array}\right.
$$




\section{4 分析与讨论}

\section{1 解释与预测}

实验结果很好地证明了以下猜想:

1) 实验结果的线性较好, 即模型在实验条件下有较高的精确度。

2) 单位体积能为负，用以降低晶体能量；单位表面能为正，用以升高晶体能量。

3) 六边形主晶面的单位表面能小 $(0.09|\alpha|)$, 而正方形次晶面的单位表面能较大 $(1.14|\alpha|)$ 。

继而可做出如下预测:

1) 当晶体足够大时主晶面将占绝对优势, 即长成正八面体。

2) 当晶体较小时, 表面能占主导地位, 晶体生长较慢; 晶体较大时, 体积能占主导地位, 晶体 生长较快。

3) 由参数 $\frac{\beta_{1}}{\alpha} 、 \frac{\beta_{2}}{\alpha}$ 可以对晶体生长或物理缺陷修补做进一步半定量的预测, 在此讨论物理缺陷 的自修复:

假设完美八面体晶体在物理作用下缺损, 在此近似为在足够大的晶体上截角正方形, 并认为除 缺陷外的其余部分动力学稳定, 在缺陷表面增厚 $\mathrm{d} h$ :

$$
\begin{aligned}
& \mathrm{d} V=b^{2} \mathrm{~d} h \\
& \mathrm{~d} S_{1}=2 \sqrt{6} b \mathrm{~d} h \\
& \mathrm{~d} S_{2}=-2 \sqrt{2} \mathrm{~d} h
\end{aligned}
$$

则对应能量变化为:

$$
\frac{\mathrm{d} E}{\mathrm{~d} h}=\alpha \frac{\mathrm{d} V}{\mathrm{~d} h}+\beta_{1} \frac{\mathrm{d} S_{1}}{\mathrm{~d} h}+\beta_{2} \frac{\mathrm{d} S_{2}}{\mathrm{~d} h}=\alpha b^{2}+\left(2 \sqrt{6} \beta_{1}-2 \sqrt{2} \beta_{2}\right) b \leq 0
$$

将参数 $\frac{\beta_{1}}{\alpha} 、 \frac{\beta_{2}}{\alpha}$ 代入, 得:

$$
b^{2}+3.78 b \geq 0
$$

即对于任意 $b \geq 0$, 都有 $\frac{\mathrm{d} E}{\mathrm{~d} h} \leq 0$, 说明晶体将自发地生成正八面体, 即明矾晶体具有自范性。

但如果对于特定晶体, 解得 $b^{2}+t b \geq 0, t<0$, 则晶体在一定尺度下将自发地出现缺陷, 如胆矾 晶体在一定大小下总生成 $(6+2 n)$ 面体。

\section{2 结果与讨论}

1) 本实验理论模型极为简化, 导致实验结果的适用精度范围较小, 而对晶体表面粗略地以 Gibbs 表面能模型处理亦可能引起误差。

2) 在本小组实验中未出现截棱的情况, 但在别的小组的实验结果中有出现单截棱的明矾晶体 (图 5)。

对单截棱的情况进行建模:

设晶体的其他部分截角与面达到热力学平衡, 截棱时 $S_{1} 、 S_{2}$ 减小使得能量降低, 但体积 $V$ 减小, 以及出现新的截棱面 $S_{3}$, 使得能量升高。当降低的能量较升高的能量多时, 出现截棱将具有热力学 优势。

如图 6 所示, 取参数 $c$, 记截去棱的高度(完美八面体的棱的截棱面的距离)为 $h$, 通过 $a 、 b 、 c$ 及设定的热力学参数可表示出现截棱后系统能量的变化。

$$
\begin{aligned}
& \Delta V=\int_{0}^{\frac{c}{2}} 2 \sqrt{2} h(a-2 b+c) \mathrm{d} h=\frac{\sqrt{2}}{6} c^{3}+\frac{\sqrt{2}}{4} c^{2}(a-2 b) \\
& \Delta S_{1}=\frac{\sqrt{3}}{2} c\left(a-2 b+\frac{c}{2}\right)=\frac{\sqrt{3}}{4} c^{2}+\frac{\sqrt{3}}{2} c(a-2 b) \\
& \Delta S_{2}=c^{2}
\end{aligned}
$$




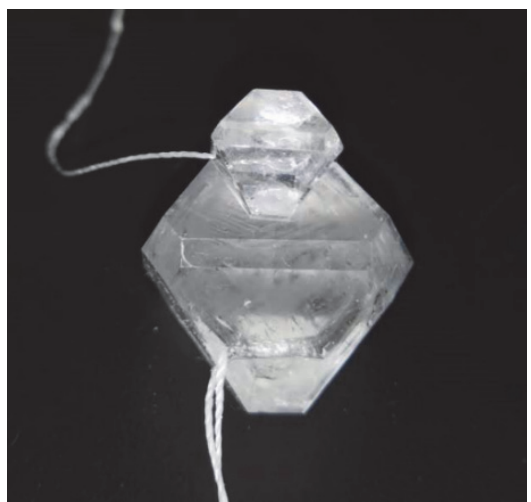

图 5 出现截棱的明矾晶体

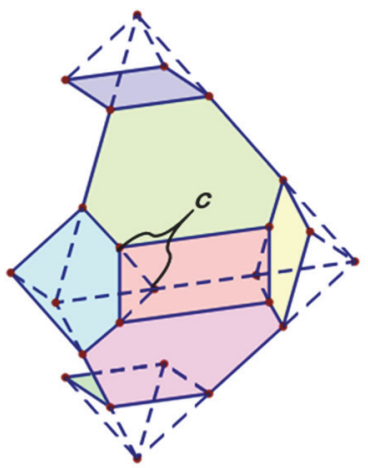

图 6 参数 $c(\mathrm{~cm})$

$$
\begin{aligned}
& \Delta S_{3}=\sqrt{2} c(a-2 b+c)=\sqrt{2} c^{2}+\sqrt{2} c(a-2 b) \\
& \Delta E=\beta_{3} \Delta S_{3}-\left(\alpha \Delta V+\beta_{1} \Delta S_{1}+\beta_{2} \Delta S_{2}\right)<0 \\
& \frac{\beta_{3}}{\alpha} \Delta S_{3}-\left(\Delta V+\frac{\beta_{1}}{\alpha} \Delta S_{1}+\frac{\beta_{2}}{\alpha} \Delta S_{2}\right)>0
\end{aligned}
$$

而截棱的晶面的表面能较高, 故应有:

$$
\left|\frac{\beta_{3}}{\alpha}\right|>\left|\frac{\beta_{2}}{\alpha}\right|>\left|\frac{\beta_{1}}{\alpha}\right|
$$

解得当 $(a-2 b)$ 较大时, $\Delta E$ 可能小于 0 使得系统出现截棱具有热力学优势 (如图 4 中下部的晶体); 而 $(a-2 b)$ 较小时亦可能由于动力学原因而出现截棱(如图 4 中上部的晶体)。

3 ) 本实验中始终将宏观明矾认为是 $O h$ 点群, 实际培养中有一些明矾为 $D_{3 d}$ 点群。

4) 建模时认为[100]面与[111]面的生长速度相等, 但实际情况中其速度应不等, 尤其当晶体较大 时，[111]面长速将远小于[100]面(即缺角的自范)。故本模型仅对较小的明矾晶体有预测性。

\section{5 结语}

上述实验操作与数据处理都较为简单，与本科生无机实验五水合硫酸铜晶体培养 ${ }^{[4]}$ 及其探究实 验明矾大晶体培养相辅相成, 在激发学生实验兴趣之余, 亦可使其通过自己的实验数据处理结合一 些结构化学与物理化学的基础知识, 解释晶体生长方面的问题, 加深对相关知识的理解, 提高科学 素养。

致谢：感谢清华大学化学系寇会忠老师对本文提供的支持和帮助。

\section{参 考 文 献}

[1] 周公度, 段连运. 结构化学基础. 第4版. 北京: 北京大学出版社, 2008: 251.

[2] 张靖阳, 蔡东龙, 刘豫健, 崔爱莉. 大学化学, 2018, 33 (12), 56.

[3] FIZ Karlsruhe. Leibniz Institute for Information Infrastructure. (2007-08-01) [2015-08-01]. https://icsd.fiz-karlsruhe.de/display/details.xhtml.

[4] 崔爱莉. 基础无机化学实验. 北京: 清华大学出版社, 2018: 71-74. 